\title{
Cuantificación de los niveles de actividad física a través de podómetros en las clases de Educación Física: Un estudio piloto
}

\author{
Pedometer-measured physical activity patterns in \\ physical education classes: A pilot study
}

\author{
María Sánchez-Baño, Andrea Visiedo y Pilar Sainz de Baranda* \\ Facultad de Ciencias del Deporte, Universidad de Murcia, Campus de Excelencia Internacional Regional "Campus Mare Nostrum".
}

\begin{abstract}
Resumen: La inactividad física durante los primeros años de vida está reconocida actualmente como un factor importante en el incremento de los niveles de obesidad y de otros trastornos médicos graves que se observan en los escolares europeos. Los escolares pasan una gran parte de su tiempo en el entorno escolar y éste debería convertirse en un entorno propicio para promocionar la actividad física. A nivel mundial, las pautas de actividad física para la salud pública incluyen un énfasis especial en poblaciones de niños (típicamente 6-11 años) y adolescentes (típicamente 12-19 años). Las directrices existentes normalmente se expresan en términos de frecuencia, el tiempo y la intensidad de la conducta. Sin embargo, con el avance tecnológico han aparecido nuevas alternativas para medir los niveles de actividad física, destacando el uso de los acelerómetros y podómetros en la investigación y en la práctica como una aproximación razonable del volumen de la actividad física diaria. El presente estudio tiene como objetivo medir la cantidad de actividad física realizada en las clases de educación física. La muestra quedó compuesta por 70 escolares (41 nińos y 29 nińas) con un rango de edad de 10 y 12 ańos en una escuela de la Región de Murcia. Un podómetro fue utilizado para cuantificar los niveles de actividad física. Se analizaron dos sesiones de la Unidad Didáctica Juegos y Deportes Alternativos. Los resultados mostraron una media de pasos/día de 3.318 (3.467,3 pasos/día en niños y 3.107,2 pasos/día en niñas). Esta cantidad representa el $20.1 \%$ y el $28.85 \%$ de la cantidad total recomendada en función del límite del rango tenido en cuenta (11.000 y 16.500 pasos/día).
\end{abstract}

Palabras claves: podómetro, educación física, promoción de la salud. Abstract: Physical inactivity during the early years of life is currently indicated as a major contributor to increased levels of obesity and other serious medical conditions and is being seen in children and adolescents all across Europe. Children spend a large part of their time at school and the school curriculum should provide good opportunities to promote physical activity. Worldwide, public health physical activity guidelines include special emphasis on populations of children (typically 6-11 years) and adolescents (typically 12-19 years). Existing guidelines are commonly expressed in terms of frequency, time, and intensity of behavior. However, the simple step output from both accelerometers and pedometers is gaining increased credibility in research and practice as a reasonable approximation of daily ambulatory physical activity volume. The present study aims to measure the amount of physical activity that takes place during physical education classes. The study comprised a sample of 70 children ( 41 boys and 29 girls) ages 10-12 from a school in Murcia (Spain). A pedometer was used to measure physical activity. Two session of learning unit titled "Games and Alternative Sports" was analyzed. The results showed a steps/day average of 3.318 (3.467,3 steps/day for boys and 3.107,2 steps/day for girls). This amount represents the $20.1 \%$ to $28.85 \%$ of the daily recommended steps/day ( 11.000 and 16.500 for minimum and maximal, respectively).

Keywords: pedometer, physical education, health promotion.

\section{Introducción}

Las repercusiones sobre la salud derivadas de la falta de actividad física (AF) ocupan un lugar preferente dentro de los problemas de salud pública, tanto en países desarrollados como en vías de desarrollo (Guthold, Ono, Strong, Chatterji y Marabia, 2008). Este problema se agrava especialmente en niños y adolescentes, quienes en las dos últimas décadas han sufrido un deterioro progresivo de su salud (hipertensión, diabetes tipo II, dislipidemia, síndrome metabólico...) debido al incremento de su peso, al aumento del sedentarismo y a la disminución de los niveles de práctica de actividad física (Huang, Ball y Franks, 2007).

Abundante literatura científica avala la relación de la prác-

Dirección para correspondencia [Correspodence address]: Pilar Sainz de Baranda. Facultad de Ciencias del Deporte. C/Argentina s/n, 30720. Santiago de la Ribera-San Javier (Murcia).

E-mail: psainzdebaranda@um.es tica de actividad física regular con la salud. Según Sallis y Patrick (1994), están establecidos los beneficios que aporta la práctica regular de actividad física a todas las edades, ya que se asocia con mejoras en numerosos aspectos fisiológicos y psicológicos.

En este mismo sentido, y desde un punto de vista preventivo, la Organización Mundial de la Salud (OMS) establece como objetivo en la lucha contra la epidemia de la obesidad infantil, lograr un equilibrio energético que se pueda mantener a lo largo de la vida del individuo, en donde se da énfasis a la importancia de que los niños acumulen al menos 60 minutos de actividad física diaria de intensidad moderada a vigorosa (AFMV).

De este modo, la comunidad científica ha centrado parte de sus estudios en describir los efectos beneficiosos de la práctica de actividad física durante todo el ciclo vital, considerando que un estilo de vida activo en edades tempranas reduce 
el riesgo de tener problemas de salud en la edad adulta (Sallis y Patrick, 1994).

Una revisión reciente apoya el efecto protector de la AF habitual contra la obesidad en la niñez y adolescencia (Jiménez-Pavón, Kelly y Reilly, 2010), no obstante, se ha demostrado que a medida que avanza la edad de los niños la práctica de AF disminuye (López Sánchez, González Víllora y Díaz Suárez, 2016; Nader, Bradley, Houts, McRitchie y O’Brien, 2008) y por tanto se hace imprescindible intervenir para paliar este descenso (Duncan, Duncan y Schofield, 2008; López Sánchez, Ahmed y Díaz Suárez, 2017).

En este contexto, las directrices de salud pública de todo el mundo están señalando con un énfasis especial la importancia de la realización, de un mínimo y con una intensidad específica, de actividad física en todas las etapas: niñez (por lo general 6-11 años), adolescencia (12-19 años) (U.S. Department of Health and Human Services, 2008; Public Health Agency of Canada y Canadian Society for Exercise Physiology, 2002) y cada vez con más interés la etapa preescolar (Timmons, Naylor y Pfeiffer, 2007).

Diversas instituciones como la OMS (2010), han elaborado recomendaciones sobre la actividad física para la salud con el objetivo general de proporcionar a los formuladores de políticas, a nivel nacional y regional, orientación sobre la relación dosis-respuesta entre frecuencia, duración, intensidad, tipo y cantidad total de actividad física.

Las directrices existentes se expresan normalmente en términos de tipo de actividad, frecuencia, tiempo e intensidad de la conducta. Sin embargo, con el avance tecnológico han aparecido nuevas alternativas para medir los niveles de actividad física, y como consecuencia nuevos parámetros para realizar las recomendaciones de práctica de actividad física, estamos hablando del número de pasos.

Con la llegada de los podómetros y los acelerómetros el potencial para estudiar los patrones complejos relacionados con la actividad física es mayor que con los medios anteriores. De estos dos instrumentos, los podómetros son más utilizados para estudios clínicos o de salud pública. Aunque también están siendo acogidos por el público en general, debido principalmente a su bajo coste y la facilidad para la interpretación de los datos (Tudor-Locke et al. 2011).

Con relación al número de pasos al día, las recomendaciones para nińos y adolescentes señalan el rango de 11.00016.500 pasos/días como la cantidad recomendada (Beets et al., 2010; Tudor-Locke et al., 2011).

Cuatro son los contextos en los que el escolar puede conseguir aumentar los niveles de actividad física a lo largo del día, 2 de ellos dentro del contexto educativo obligatorio: 1) Clases de Educación Física y 2) Recreos; y otros 2 fuera del contexto educativo obligatorio: 3) Clases de actividad extraescolar y 4) Tiempo libre.

El objetivo del presente trabajo es monitorizar el número de pasos por sesión que dan los alumnos/as en las clases de Educación Física y comprobar que parte de la recomendación pasos/día se cumple.

\section{Material y método}

\subsection{Participantes}

Muestra de conveniencia de 70 escolares del tercer ciclo de

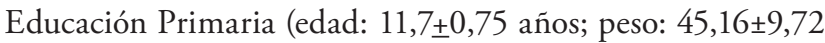
Kg; talla: $149,6 \pm 7,91 \mathrm{~cm})$. De cada curso se seleccionaron dos clases, $5^{\circ} \mathrm{A}$ y $5^{\circ} \mathrm{B}$ y $6^{\circ} \mathrm{A}$ y $6^{\circ} \mathrm{B}$. El grupo $5^{\circ} \mathrm{A}$ estaba formado por 18 alumnos (13 niños y 5 niñas), el grupo $5^{\circ} \mathrm{B}$ por 15 alumnos (10 niños y 5 niñas), el grupo 6\% $6^{\circ}$ por 18 alumnos (7 niños y 11 niñas) y el grupo $6^{\circ} \mathrm{B}$ por 19 alumnos (10 niños y 9 nińas). Las edades de los alumnos estaban comprendidas entre los 10 y los 12 ańos.

Como criterios de exclusión se establecieron: (a) no asistir a alguna de las sesiones de Educación Física. Los progenitores y/o tutores fueron verbalmente informados de la metodología a utilizar, así como de los propósitos del estudio, y un consentimiento informado fue firmado por cada uno de ellos. El presente estudio fue aprobado por el Comité Ético y Científico de la Universidad de Murcia (España).

\subsection{Procedimiento}

El estudio se llevó a cabo durante el curso académico 20132014. En primer lugar y como rutina habitual se recogió en la Ficha de Salud del alumno el peso y la talla. Posteriormente, durante la última semana del mes de mayo de 2014, se llevó a cabo la toma de datos utilizando para ello las sesiones correspondientes a la Unidad Didáctica "Juegos y deportes alternativos" (anexo I y II). Para la cuantificación del número de pasos se utilizaron 70 podómetros DN100 (Oxylane, Villeneuve d'Ascq cedex, Francia). Los podómetros iban marcados con un número y el monitor tapado, ese número pertenecía a un alumno/a concreto. De esta forma no había que modificar los datos de los podómetros, ya que se introdujeron talla y peso de cada nińo/a.

Los podómetros se colocaron en clase, en el momento en el que el maestro de Educación Física recogía a los alumnos para ir a la pista polideportiva. De este modo la sesión quedaba organizada de la siguiente manera:

- Parte inicial o calentamiento: los alumnos/as con los podómetros colocados y de forma autónoma realizaron el calentamiento y movilidad articular. Duración de 7 minutos.

- Parte principal: en esta parte de la sesión se llevó a cabo el objetivo de la Unidad Didáctica programada: "Juegos y deportes alternativos", con una duración de 45 minutos (anexo I y II). 
- Parte final o vuelta a calma: Se realizan los estiramientos, se comenta la sesión y se realiza el aseo personal. Una vez finalizada se anotaron los datos arrojados por los podómetros. Duración 5 minutos.

Cuando los alumnos finalizaban la clase, el profesor registraba el número de pasos de cada podómetro y los retiraba. Se indicó a los escolares que no debía tocar el podómetro en ningún momento

El podómetro DN100 fue seleccionado para la presente investigación por ser económico y accesible. Además, sus características en tamaño y fijación lo hacían preferible para su utilización con escolares.

Una reciente revisión publicada por McNamara, Hudson y Taylor (2010) recoge 25 estudios que evalúan la validez y fiabilidad de los podómetros en niños, poniendo de manifiesto que pueden ser utilizados como instrumentos válidos y fiables para determinar los niveles de actividad física en este colectivo. Específicamente, la validez del podómetro El DN100 ha sido analizada en el estudio de López-Fernández, Pascual-Martos y Álvarez-Carnero (2013).

\subsection{Análisis estadístico}

En primer lugar se realizó un estudio descriptivo utilizando la media, desviación típica, mínimo, máximo, así como análisis de frecuencias. En segundo lugar, y con el objetivo de poder comparar los valores medios entre las diferentes variables objeto de estudio, se comprobó con la normalidad de los datos y se utilizó la prueba $\mathrm{T}$ para muestras independientes. Para el análisis estadístico de los datos se utilizó el paquete estadístico SPSS 20.0.

\section{Resultados}

La media del número de pasos registrados en las dos sesiones fue de $3.467,3 \pm 805,56$. En la tabla 1 se aprecia los valores medios mínimo y máximo del número de pasos que los escolares realizaron en las dos sesiones analizadas. El análisis estadístico refleja que los niños realizaron una media mayor de pasos, aunque no se apreciaron diferencias estadísticamente significativas en ninguna variable analizada: pasos sesión 1 $\left(\mathrm{t}_{68}=1.807, \mathrm{p}=.075\right)$, pasos sesión $2\left(\mathrm{t}_{68}=1.924, \mathrm{p}=.058\right)$ y pasos media $\left(\mathrm{t}_{68}=1.906, \mathrm{p}=.061\right)$.

Tabla 1. Valores medios de pasos realizados en la unidad didáctica "Juegos y deportes alternativos".

\begin{tabular}{cccccc}
\hline Sesión & Nińo & Niña & Total & Mínimo & Máximo \\
\hline Sesión 1 & $3.384 \pm 786$ & $3.052 \pm 709$ & $3.246,9 \pm 768$ & 1.921 & 4.879 \\
Sesión 2 & $3.550 \pm 869$ & $3.161 \pm 777$ & $3.389,2 \pm 848$ & 1.898 & 5.654 \\
Media & $3.467 \pm 805$ & $3.107 \pm 738$ & $3.318,2 \pm 793$ & 1.909 & 5.266 \\
\hline
\end{tabular}

\section{Discusión}

El ámbito escolar es un entorno adecuado para la influencia y obtención de hábitos saludables en cuanto a la práctica deportiva. Es tarea de los maestros y profesores de Educación Física, incentivar la práctica y transmitir los beneficios de la misma, ya que la escuela se convierte en algunas ocasiones, en el único lugar donde los alumnos/as tienen la posibilidad de realizarla. Diversos estudios han concluido que la clase de Educación Física constituye el único tiempo en el que la mitad de la población infantil realiza algún tipo de actividad física (Hernández y Velázquez, 2007).

En este sentido, el grado de satisfacción con las clases de Educación Física y su calidad, son factores influyentes en la adherencia a la actividad física y en la adopción de un estilo de vida activo y saludable (Stelzer, Ernest, Fenster y Langford, 2004).

Ante estos resultados, algunos países han optado por centrar su atención en el área de Educación Física y también en la escuela, señalando a ambas dentro de las directrices que van en beneficio de la población escolar. Como ejemplo de esta iniciativa, en Estados Unidos se ha incluido como objetivo de salud para la nación, metas que promueven la actividad física de intensidad moderada a vigorosa en las clases de Educación Física, recomendando diariamente la realización de una AFMV durante el 50\% de la sesión de Educación Física (US Department of Health and Human Services, 2000).

El presente trabajo de investigación se ha centrado en valorar los niveles de práctica de actividad física que se pueden conseguir en las sesiones de Educación Física, de una unidad didáctica relacionada con "los Juegos y Deportes Alternativos”, ya que como afirman Tudor-Locke, McClain, Hart, Sisson y Washington (2009): "Los datos descriptivos son necesarios para ayudar en la comparación e interpretación de datos similares que analicen los niveles de actividad física a través del número de pasos".

Tras el análisis de los resultados, se ha encontrado una media de 3.318,2 pasos/sesión, con un valor mínimo de 1.910 pasos/sesión y un máximo de 5.267 pasos/sesión. Estos resultados, representan el 28,85\% del número de pasos que un escolar debe realizar durante un día si se tiene en cuenta el límite inferior de la recomendación (11.000 pasos/días) y el $20,1 \%$ si se tiene en cuenta el límite superior de la recomendación (16.500 pasos/días). 
En la literatura internacional se pueden encontrar estudios que han valorado el número de pasos realizados durante el horario escolar, tanto en escolares estadounidenses como en escolares del resto de países. Así, tras el análisis de 30 estudios, en una revisión realizada por Brusseau y Hannon (2013), se observa como dentro del horario escolar, las nińas estadounidenses dan de promedio entre 2.500-5.500 pasos y los niños estadounidenses entre 3.800-6.800 pasos. Mientras que los escolares de otros países del mundo dan de promedio entre los 3.000 y 7.000 pasos y los 3.500-8.100 pasos para niñas y niños, respectivamente.

Cuando se comparan estos datos con los del presente estudio, se observa que los 3.318,2 pasos por sesión realizados de media durante la sesión de Educación Física superan el número de pasos que un escolar realiza durante una jornada escolar, si se tiene en cuenta el límite inferior observado en los distintos estudios (3.000 pasos/jornada escolar) y representan el $40,9 \%$ de los pasos que da un escolar en una jornada escolar, si se tiene en cuenta el límite superior de la recomendación (8.100 pasos/jornada escolar).

Indudablemente, los pasos realizados en la sesión de Educación Física no son suficientes para cumplir las recomendaciones de pasos/día. Por ello, es necesario reflexionar y plantear varias preguntas: ¿Se podrían llegar a cumplir las recomendaciones de actividad física al día sólo con la clase de Educación Física? ¿Sería obligatorio para ello aumentar la duración de la misma? ¿Los datos encontrados en este estudio son buenos o son insuficientes? ¿Podría estar el número de pasos condicionado por el contenido a trabajar o por la metodología empleada?

Lo que es evidente, es que el maestro o profesor de Educación Física debe tener en cuenta este dato para reflexionar sobre sus clases. Además, y como agente promotor de salud, debería informar a los alumnos y sus progenitores de la cantidad restante de actividad física que el escolar debe realizar durante el resto del día. Por otro lado, y dentro en este caso del contexto escolar, el maestro o profesor de Educación Física debe tener en cuenta el recreo como otro elemento clave para aumentar los niveles de actividad física de los escolares.

Algunos estudios como el de López-Fernández et al. (2013) han analizado el número de pasos que se realizan durante un recreo de 30 minutos, encontrando una media de $2.539 \pm 991,11$ pasos.

Martínez, Aznar y Contreras (2015), utilizando acelerómetros para valorar los niveles de actividad física en los recreos escolares, observan cómo los 30 minutos de los que disponen los alumnos/as de tiempo de ocio, pueden colaborar a aumentar la AF saludable del día. En este mismo sentido, Ridgers et al. (2011) mostraron como el recreo contribuía aproximadamente al 16\% del total de la AF saludable del día. Ambos estudios, concluyen que el recreo dentro del contexto no curricular adquiere un papel importante, idea que lo sitúa en el punto de partida para futuras intervenciones.

De forma general, ambos datos, tanto los relacionados con la sesión de Educación Física, como los relacionados con el recreo son importantes, ya que los pasos totales acumulados durante el horario escolar representan del 35\% al 46\% del total de la actividad física realizada para las niñas y del $35 \%$ al 53\% para los nińos (Brusseau y Hannon, 2013).

Con relación a la intensidad a la que se debe realizar la actividad física, las recomendaciones indican que los niños/as y adolescentes deben realizar al menos 60 minutos de actividad física de intensidad moderada a vigorosa, todos o la mayoría de los días de la semana. De igual modo la Asociación Americana del Corazón (Pate et al., 2006), subraya que al menos la mitad de las recomendaciones diarias (30 min de AFMV) deberían ser realizadas dentro del currículo escolar.

Los estudios controlados de observación han encontrado que caminar de forma continua a una intensidad de moderada a vigorosa produce de 3.300 a 3.500 pasos en 30 minutos o 6.600-7.000 pasos en 60 minutos en escolares de 10-15 ańos. La evidencia limitada sugiere que un volumen diario total de actividad física de 10.000-14.000 pasos/día se asocia a 60100 minutos de AFMV en niños de preescolar (de aproximadamente 4-6 años de edad).

Por otro lado, los estudios realizados con escolares de primaria han observado que en 60 minutos de actividad física de intensidad moderada a vigorosa se alcanza un promedio, dentro de un volumen total de 13.000 a 15.000 pasos/día en niños y 11.000 a 12.000 pasos/día en las nińas. Para los adolescentes (chicos y chicas), un rango entre 10.000 y 11.700 pasos/día puede estar asociados con 60 minutos de actividad física de intensidad moderada a vigorosa.

Partiendo de los resultados del presente estudio, si los escolares realizan de media 3.318,2 pasos en 55 minutos, es evidente que no se consigue realizar una AFMV suficiente en la sesión.

Por tanto, y siguiendo a autores como Martínez, Contreras, Lera y Aznar (2012), se puede concluir que la proporción de tiempo de AFMV en las sesiones de Educación Física es bajo. En su investigación usando acelerómetros, los alumnos de una clase de $3^{\circ}$ ciclo de Primaria, con edades comprendidas entre los 10 a 12 ańos, realizaron 16,6 minutos de AFMV en una clase de 120 minutos, lo que corresponde al 9,3\% de la sesión.

Tras los resultados relacionados con la intensidad de la práctica alcanzada en las clases de Educación Física, es necesario de nuevo reflexionar y plantear varias preguntas: ¿ $\mathrm{Se}$ pueden llegar a cumplir las recomendaciones de intensidad de actividad física al día en la clase de Educación Física? ¿Podría estar condicionado por el contenido a trabajar o por la metodología empleada? ¿Qué papel puede tener el maestro o profesor? ¿Pueden influir otros factores como el sexo, la edad, el nivel de obesidad o el nivel de condición física? 
Este estudio tiene varias limitaciones, siendo la más importante el hecho de que la monitorización se realizó durante solo dos sesiones, por lo tanto los resultados no pueden generalizarse a la calidad promedio de las clases.

En futuros trabajos, sería importante realizar esta monitorización en varios momentos del curso escolar, abordando distintos objetivos y contenidos curriculares, investigando si existe más o menos AFMV dependiendo del tipo de contenido trabajado (habilidades y destrezas motrices, juegos y deportes, coordinación y equilibrio, esquema corporal, actividades artístico-expresivas, actividad física y salud).

De interés sería extrapolar este estudio a todos los cursos, desde $1^{\circ}$ de Educación Primaria hasta $6^{\circ}$, para analizar las edades comprendidas de los 6 a los 8 años, y de los 8 a los 10. Igualmente, sería conveniente cuantificar el número de pasos que darían los escolares en el nuevo planteamiento de la Consejería de Educación de la Región de Murcia, de llevar a cabo el Plan de Fomento de la Actividad Física y la Salud, reflejado en los recreos escolares, planteando que éstos sean activos y saludables. Analizar cuántos pasos realizan los alumnos/as durante la hora y media semanal de este tiempo de permanencia en el centro, añadiéndola a la actividad física que realizan en las sesiones y comprobar si se siguen las directrices saludables.

Otro aspecto de futuras investigaciones, sería determinar la influencia de la labor docente (talante, empatía con los alumnos/as, metodologías utilizadas,...) como factor determinante en la variación de la AFMV en sus alumnos/as.

\section{Conclusiones}

Este estudio demuestra que de promedio en una sesión de Educación Física relacionada los "Juegos y Deportes alternativos" los escolares realizan 3.318 pasos, lo cual representa entre el $20.1 \%$ y el $28.85 \%$ de la cantidad total de actividad física que deben realizar a lo largo del día, cuando se tiene en cuenta la recomendación de hacer un rango de pasos entre los 11.000 y los 16.500 pasos.

\section{Bibliografía}

1. Brusseau, T.A. y Hannon, J.C. (2013). Pedometer-Determined Physical Activity of Youth while Attending School: A Review. Sport Science Review, 22(5-6), 329-342.

2. Duncan, E.K., Duncan, J. S. y Schofield, G. (2008). Pedometer-determined physical activity and active transport in girls. International Journal of Behavioral Nutrition and Physical Activity, 5(1), 2.

3. Guthold, R., Ono, T., Strong, K. L., Chatterji, S. y Morabia, A. (2008). Worldwide variability in physical inactivity: a 51 -country survey. American Journal of Preventive Medicine, 34(6), 486-494.

4. Hernández, J.L. y Velázquez, D.A. (2007). La educación física, los estilos de vida y los adolescentes: Cómo son, cómo se ven, qué saben y qué opinan (Vol. 242). Barcelona: Graó.

5. Huang, T.T.K., Ball, G.D. y Franks, P.W. (2007). Metabolic syndrome in youth: current issues and challenges. Applied Physiology, Nutrition, and Metabolism, 32(1), 13-22.

6. Jiménez-Pavón, D., Kelly, J. y Reilly, J.J. (2010). Associations between objectively measured habitual physical activity and adiposity in children and adolescents: Systematic review. International Journal of Pediatric Obesity, 5(1), 3-18.

7. López-Fernández, I., Pascual-Martos, C. A. y Álvarez-Carnero, E. (2013). Validación de un podómetro para medir la actividad física en los recreos escolares. Journal of Sport and Health Research, 5(2), 167-178.

8. López Sánchez, G. F., González Víllora, S. \& Díaz Suárez, A. (2016). Level of habitual physical activity in children and adolescents from the Region of Murcia (Spain). SpringerPlus, 5:386, 1-6. DOI 10.1186/ s40064-016-2033-8

9. López Sánchez, G. F., Ahmed, D., Díaz Suárez, A. (2017). Level of habitual physical activity among 13-year-old adolescents from Spain and India. A cross-cultural study. SPORT TK: Revista Euroamericana de Ciencias del Deporte, 6(1),67-74.

10. McNamara, E., Hudson, Z. y Taylor, SJ. (2010). Measuring activity levels of Young people: the validity of pedometers. British Medical Bulletin, 95, 121-137.

11. Martínez, J., Aznar, S. y Contreras, O.R. (2015). El recreo escolar como oportunidad de espacio y tiempo saludable. Revista Internacional de Medicina y Ciencias de la Actividad Física y del Deporte, 15(59), 419-432.
12. Martínez, J., Contreras, O. R., Lera, Á. y Aznar, S. (2012). Niveles de actividad física medido con acelerómetro en alumnos de $3^{\circ}$ ciclo de educación primaria: actividad física diaria y sesiones de educación física. Revista de Psicología del Deporte, 21(1), 117-123.

13. Nader, P. R., Bradley, R. H., Houts, R. M., McRitchie, S. L. y O'Brien, M. (2008). Moderate-to-vigorous physical activity from ages 9 to 15 years. JAMA, 300(3), 295-305.

14. OMS. (2010). Recomendaciones mundiales sobre actividad fisica para la salud. Geneva: WHO Library Cataloguing-in-Publication Data.

15. Pate, R.R., Davis, M.G., Robinson, T.N., Stone, E.J., McKenzie, T.L., Young, J.C., American Heart Association Council on Nutrition, Physical Activity, and Metabolism (Physical Activity Committee), Council on Cardiovascular Disease in the Young; Council on Cardiovascular Nursing. (2006). Promoting physical activity in children and youth: a leadership role for schools: a scientific statement from the American Heart Association Council on Nutrition, Physical Activity, and Metabolism. Circulation, 114(11),1214-1224.

16. Public Health Agency of Canada \& Canadian Society for Exercise Physiology. (2002a). Canada's Physical Activity Guide to Healthy Active Living for Children. Ottawa, Ont.: Public Health Agency.

17. Public Health Agency of Canada \& Canadian Society for Exercise Physiology. (2002b). Canada's Physical Activity Guide to Healthy Active Living for Youth. Ottawa, Ont.: Public Health Agency.

18. Ridgers, N.D., Saint-Maurice, P.F., Welk, G.J., Siahpush, M. y Huberty, J. (2011). Differences in physical activity during school recess. Journal of School Health, 81(9), 545-551.

19. Sallis, J.F. y Patrick, K. (1994). Physical activity guidelines for adolescents: consensus statement. Pediatric Exercise Science, 6 (4), 302-314.

20. Stelzer, J., Ernest, J.M., Fenster, M.J. y Langford, G. (2004). Attitudes toward Physical Education: A Study of High School Students from Four Countries-Austria, Czech Republic, England, and USA. College Student Journal, 38(2), 171.

21. Timmons, B.W., Naylor, P.J., y Pfeiffer, K.A. (2007). Physical activity for preschool children--how much and how?. Canadian Journal of Public Health, 98(Suppl 2), S122-134.

22. Tudor-Locke, C., Craig, C.L., Beets, M.W., Belton, S., Cardon, G.M., 
Duncan, S. y Blair, S.N. (2011). How many steps/day are enough? For children and adolescents. International Journal of Behavioural Nutrition and Physical Activity. 8 (78).

23. Tudor-Locke, C., McClain, J.J., Hart, T.L., Sisson, S.B., y Washington, T.L. (2009). Pedometry methods for assessing free-living youth. Research Quarterly for Exercise and Sport, 80(2), 175-184.
24. U.S. Department of Health and Human Services. (2008) Physical Activity Guidelines for Americans: Be Active, Healthy, and Happy! Washington, D.C.

25. US Department of Health and Human Services USDHHS, EEUU, 2000. Disponible en http://www.hhs.gov/ 







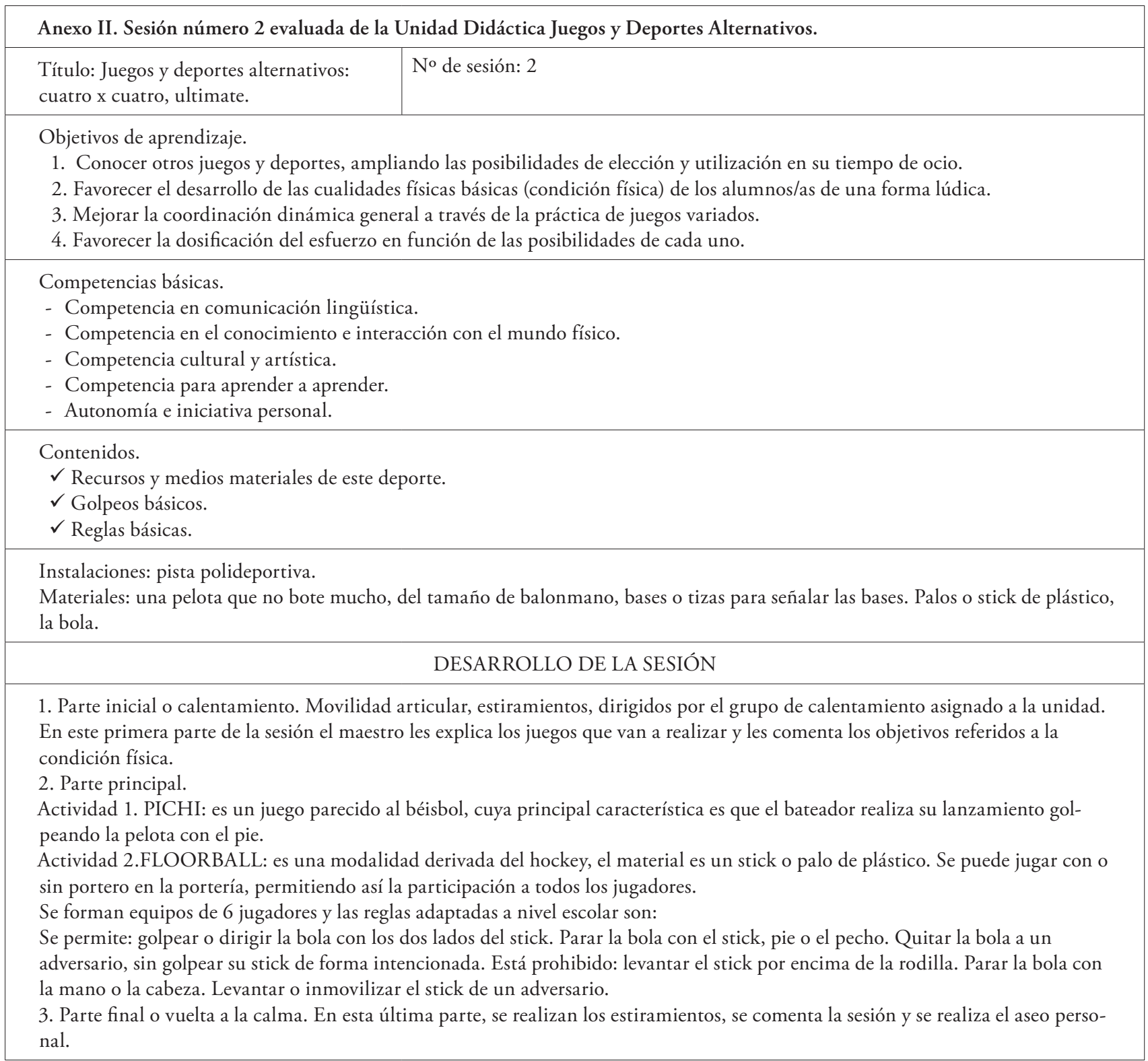

